\title{
Correction to: Study of Transition Metal Ion Doped CdS Nanoparticles for Removal of Dye from Textile Wastewater
}

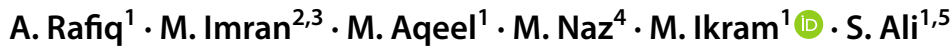

Published online: 14 November 2019

○) Springer Science+Business Media, LLC, part of Springer Nature 2019

\section{Correction to: \\ Journal of Inorganic and Organometallic Polymers and Materials \\ https://doi.org/10.1007/s10904-019-01343-5}

The original version of this article unfortunately contained few mistakes. In Fig. 7 caption, Co:ZnS should be replaced with Co:CdS and Fe:ZnS should be replaced with Fe:CdS. In Table 1, the column heading TM doped $\mathrm{ZnS}$ should be replaced with TM doped CdS. In Table 1, the last word under the column heading Photocatalysis should read as $\mathrm{CdS}$ instead of $\mathrm{ZnS}$. In the Conclusion section, $\mathrm{ZnS}$ should be replaced with $\mathrm{CdS}$ in the seventh and tenth lines.
Publisher's Note Springer Nature remains neutral with regard to jurisdictional claims in published maps and institutional affiliations.

The original article can be found online at https://doi.org/10.1007/ s10904-019-01343-5.

M. Ikram

dr.muhammadikram@gcu.edu.pk

1 Solar Cell Applications Research Lab, Department of Physics, Government College University Lahore, Punjab 54000, Pakistan

2 Technical Institute of Physics and Chemistry, Chinese Academy of Sciences, 29 Zhongguancun East Road, Haidian District, Beijing 100190, China

3 University of Chinese Academy of Sciences, Beijing 100049 , China

4 Biochemistry Lab, Department of Chemistry, Government College University Lahore, Punjab 54000, Pakistan

5 Department of Physics, Riphah Institute of Computing and Applied Sciences (RICAS), Riphah International University, 14 Ali Road, Lahore, Pakistan 